\title{
Molecular Biomarkers and Epidemiologic Risk Assessment
}

\author{
Paul W. Brandt-Rauf, ${ }^{1 *}$ Jiin-Chyuan Luo, ${ }^{2}$ Tsun-Jen Cheng, ${ }^{3}$ Chung-Li Du, ${ }^{3}$ Jung- \\ Der Wang, ${ }^{3}$ Ramon Rosal, ${ }^{1}$ Tamara Do, ${ }^{1}$ and Marie-Jeanne Marion ${ }^{4}$ \\ ${ }^{1}$ Department of Environmental Health Sciences, The Mailman School of Public \\ Health of Columbia University, 60 Haven Avenue, New York, NY 10032, USA \\ (author for correspondence); Tel(voice):212-305-3959, Tel(fax):212-305-4012; \\ pwb1@columbia.edu. ${ }^{2}$ Department of Public Health, Chang Gung Medical \\ College, Taipei, Taiwan. ${ }^{3}$ Graduate Institute of Occupational Medicine and \\ Industrial Hygiene, National Taiwan University, Taipei, Taiwan. ${ }^{4}$ Unite de \\ Recherche sur les Virus des Hepatites et Pathologies Associees, INSERM, 69424 \\ Lyon, France
}

\begin{abstract}
The use of molecular biomarkers in epidemiologic studies has been advanced as a way to improve risk assessments for occupational and environmental exposures to toxic agents. We have used the detection of two cancer-related, molecular biomarkers of vinyl chloride exposure (mutant ras-p21 and mutant p53) to examine workers with equivalent cumulative exposures that would be above or below the current permissible workplace exposure limit for vinyl chloride for differences in the presence of these biomarkers. Workers with cumulative exposures above the current permissible exposure limit (equivalent of $>40$ ppm-years) have a statistically significantly increased occurrence of both biomarkers in comparison to unexposed controls $\left(\mathrm{p}<10^{-3}\right)$. Although workers with cumulative exposures of $<10$ ppm-years, i.e., well below the current limit, do not have a statistically significantly increased occurrence of these biomarkers $(p>0.05)$, workers with cumulative exposures of 10 to 40 ppm-years, i.e., still below the current limit, are found to have a statistically significant increase $(\mathrm{p}<0.05)$. This suggests that the current exposure limit may not be adequately protective and illustrates the potential utility of molecular biomarkers in the refinement of risk assessments for toxic exposures.
\end{abstract}

Key Words: vinyl chloride, cancer, mutations, exposure limit.

Corresponding author.

$1080-7039 / 02 / \$ .50$

(C) 2002 by ASP 


\section{INTRODUCTION}

In recent years there has been an explosion of knowledge concerning the molecular pathways by which occupational and environmental toxins produce adverse health effects. This has led to the development of molecular biomarkers that can be used to identify various steps in these pathways in vivo in exposed human populations. The use of such biomarkers has been suggested as a way to refine and enhance group and individual risk assessments for toxic occupational and environmental exposures (Hattis and Silver 1993). Rather than relying on traditional estimates of ambient exposures, categorization by clinical disease diagnoses, and stratification by crude external population risk characteristics, the use of molecular biomarkers of dose, effect, and susceptibility, respectively, should be able to provide more precise and mechanistically realistic metrics for the risk assessment process. For example, the use of molecular biomarkers of biologically effective dose (DNA and hemoglobin adducts) have been applied to the risk assessment of genotoxic alkylating agents, such as ethylene oxide (Tornqvist and Landin 1995). More recently, we have attempted to use molecular biomarkers of response (mutant oncoproteins) to examine the risk assessment for workplace exposure to vinyl chloride (VC) (Brandt-Rauf et al. 2000). VC provides a particularly good example because considerable detail is available concerning its potential mechanism of action.

$\mathrm{VC}$ is a known carcinogen that is rapidly absorbed following respiratory exposure, and it is subsequently metabolized in the liver by the cytochrome P450 2E1 system (ATSDR 1997). The resultant electrophilic metabolites, chloroethylene oxide and chloroacetaldehyde, can form a variety of DNA adducts that are known to be promutagenic, including 7-(2'-oxoethyl)guanine, 1, $N^{6}$ - ethenoadenine, 3, $N^{4}$ ethenocytosine and $N^{2}, 3$-ethenoguanine (Barbin and Bartsch 1986). Although the oxoethyl adduct accounts for the vast majority of adducts formed, it is rapidly repaired and probably does not contribute to the carcinogenic effects of VC. On the other hand, the less common etheno adducts are poorly repaired and have long half-lives accounting for the production of the specific point mutations identified in VC-related malignancies (Swenberg et al. 1992). For instance, $83 \%$ of angiosarcomas of the liver (ASL) from VC-exposed workers have been found to contain $\mathrm{G} \rightarrow \mathrm{A}$ transitions in the Ki-ras oncogene that could be attributed to the generation of ethenoguanine adducts by VC (Marion et al. 1991). Similarly, $60 \%$ of ASLs from VCexposed workers have been found to contain $\mathrm{A} \rightarrow \mathrm{T}$ transversions in the p53 tumor suppressor gene that could be attributed to the generation of ethenoadenine adducts by VC (Hollstein et al. 1994). The occurrence of each of these cancerrelated mutations leads to the production of mutant oncoprotein biomarkers (mutant ras-p21 protein and mutant p53 proteins and/or auto-antibodies to mutant p53 proteins) that can be detected in the blood of individuals who have tumors that contain the respective mutations (DeVivo et al. 1994; Brandt-Rauf et al. 1996). Furthermore, these same oncoprotein biomarkers have been identified in several cohorts of workers around the world exposed to $\mathrm{VC}$ but without known malignant disease, and they have been found to occur with a significant dose-response relationship with regard to the workers' estimated, cumulative VC exposure at levels above the equivalent current permissible exposure limit used in most western countries of 
$1 \mathrm{ppm}$ (i.e., cumulative exposures of 40 ppm-years, or 1 ppm for 40 working years) (Smith et al. 1998; Li et al. 1998; Luo et al. 1998, 1999). However, in each of these cohorts the presence of these oncoprotein biomarkers has also been detected in workers with equivalent cumulative exposures that would be below the current permissible exposure limit (i.e., below 40 ppm-years). This has recently prompted us to combine this biomarker data from the different cohorts to examine the prevalence of the biomarkers in various sub- groups of workers with equivalent cumulative exposures below the current permissible exposure limit (Brandt-Rauf et al. 2000). This allows us to reevaluate the validity of the risk assessment that forms the basis for the current exposure limit, assuming that the presence of these biomarkers represents a significant cancer risk. The specific issue to be addressed was whether there exist subgroups of workers with equivalent cumulative exposures below the current limit that have statistically significant increases in the prevalence of these VC-induced, cancer-related biomarkers.

\section{METHODS}

Two previously described cohorts of VC workers and matched, unexposed controls, one from France and one from Taiwan, were included in the analysis (Smith et al. 1998; Luo et al. 1998). The combined cohort was comprised of 468 exposed workers, 306 with cumulative exposure $>40$ ppm- years and 109 with cumulative exposure $\leq 40$ ppm-years, and 155 matched, unexposed controls. Blood samples had been collected from these individuals by routine venipuncture techniques and stored frozen until the time of analysis for the mutant ras-p21 and mutant p53 oncoprotein biomarkers. The biomarkers were analyzed as described in detail previously, relying on monoclonal antibodies that are specific for the detection of the mutant oncoproteins (DeVivo et al. 1994; Smith et al. 1998).

Logistic regression analysis was performed using the unexposed controls as reference with an assigned odds ratio of one, and odds ratios were calculated for various subgroups of exposed workers for the presence of one or both oncoprotein biomarkers with adjustment for previously identified potential confounders including age, smoking and alcohol consumption. Initially, the analysis was performed with the workers stratified into those above and those below the equivalent current permissible exposure limit of 40 ppm-years. Subsequently, the analysis was performed with the workers with equivalent cumulative exposures below the current permissible exposure limit being further stratified into those with 0-10 ppm-years, 10 to 20 ppm-years, 20 to 30 ppm-years, and 30 to 40 ppm- years. Finally, based on the results of the sub-stratification of the workers with equivalent cumulative exposures below the current permissible exposure limit, the workers were grouped into those with $<10$ ppm-years of cumulative exposure and those with 10 to 40 ppm-years of cumulative exposures, and these were compared to the workers with cumulative exposures $>40$ ppm-years and to the unexposed controls.

\section{RESULTS}

Only 16 of the 155 unexposed controls $(10 \%)$ were seropositive for one or the other of the oncoprotein biomarkers, and no unexposed control $(0 \%)$ was positive 
for both oncoprotein biomarkers. Of the $306 \mathrm{VC}$ workers with cumulative exposures $>40$ ppm-years, $122(40 \%)$ were seropositive for one or the other oncoprotein biomarker (adjusted odds ratio $=5.0, \mathrm{p}<10^{-3}$ ) and $33(11 \%)$ were seropositive for both oncoprotein biomarkers (adjusted odds ratio $=7.3, \mathrm{p}<10^{-3}$ ), both highly statistically significant differences compared to the unexposed controls. Of the 162 workers with cumulative exposures $\leq 40$ ppm-years, 33 (20\%) were seropositive for one or the other oncoprotein biomarker (adjusted odds ratio $=1.4, \mathrm{p}=0.37$ ) and $3(2 \%)$ were seropositive for both oncoprotein biomarkers (adjusted odds ratio = $4.4, \mathrm{p}=0.06$ ).

Although neither of the latter results were statistically significant compared to the unexposed controls, both odds ratios were elevated, and, in the latter case, the odds ratio approached statistical significance, suggesting perhaps that subgroups among the workers with cumulative exposures $\leq 40 \mathrm{ppm}$-years could have significantly elevated risks for the occurrence of the biomarkers, particularly when both biomarkers were considered together. Further analysis with stratification of this group into four subgroups with cumulative exposures of $<10$ ppm-years, 10 to 20 ppm-years, 20 to $30 \mathrm{ppm}$-years, and 30 to $40 \mathrm{ppm}$-years supported this assumption. For example, for the subgroup with cumulative exposures $<10$ ppm-years the adjusted odds ratios for the presence of one or both biomarkers were not greatly elevated (both $<2$ ), and neither was close to statistical significance. On the other hand, for the other three subgroups, although the adjusted odds ratios for the presence of one biomarker were similarly not greatly elevated, the adjusted odds ratios for the presence of both biomarkers were considerably higher, indicating that the increased risk for those with cumulative exposures $\leq 40 \mathrm{ppm}$-years was primarily among those workers with cumulative exposures of 10 to 40 ppm-years. Therefore, further analysis was confined to the cohort stratified by cumulative exposures of $<10$ ppm-years, 10 to 40 ppm-years and $>40$ ppm-years compared to the unexposed controls, as shown in Table 1. In this case, for the sub-group with cumulative exposures of 10 to $40 \mathrm{ppm}$ years, the adjusted odds ratio for the presence of one biomarker was only 1.2 and remained statistically insignificant $(\mathrm{p}=0.67)$. However, in this subgroup the adjusted odds ratio for the presence of both biomarkers was 5.7 and was statistically significant $(\mathrm{p}=0.045)$. In fact, this elevated risk was not significantly different from

Table 1. Relationship between molecular biomarkers of cancer-related mutations and estimated cumulative VG exposure.

\begin{tabular}{|lccccc|}
\hline $\begin{array}{c}\text { Exposure } \\
\text { in PPM-Years (N) }\end{array}$ & $\begin{array}{c}\text { Both } \\
\text { Negative }\end{array}$ & $\begin{array}{c}\text { One } \\
\text { Positive }\end{array}$ & $\begin{array}{c}\text { Both } \\
\text { Positive }\end{array}$ & OR $^{1}$ & OR $^{2}$ \\
$0(\mathrm{~N}=155)$ & 139 & 16 & 0 & 1 & 1 \\
$<10(\mathrm{~N}=77)$ & 59 & 17 & 1 & 1.9 & 1.7 \\
$10-40(\mathrm{~N}=85)$ & 67 & 16 & 2 & 1.2 & $5.7^{*}$ \\
$>40(\mathrm{~N}=306)$ & 151 & 122 & 33 & $5.0^{*}$ & $7.3^{*}$ \\
\hline
\end{tabular}

${ }^{1}$ Odds ratio for one positive vs both negative adjusted for age, smoking and drinking

${ }^{2}$ Odds ratio for both positive vs both negative adjusted for age, smoking and drinking

${ }^{*} \mathrm{p}<0.05$ 
that found for the presence of biomarkers in the group with cumulative exposures $>40$ ppm-years.

\section{DISGUSSION}

These biomarker results suggest that some workers with VC exposures below the current permissible exposure limit have a risk for the occurrence of two VCinduced, cancer-related mutations that is similar to that for workers exposed above this limit and that is significantly greater than unexposed controls. Although we do not know at this time whether these individuals positive for the biomarkers will actually develop cancer, results from studies of other exposed cohorts of workers using banked serum samples suggest that similar biomarkers can have considerable positive predictive value (Husgafvel-Pursiainen et al. 1997). If the same proves to be true in these VC workers, this would mean that workers with cumulative exposures of 10 to 40 ppm-years (just like workers with cumulative exposures $>40$ ppm-years) could be at an increased risk for cancer and that the current permissible exposure limit is not adequately protective. In addition, since workers with cumulative exposures $<10$ ppm-years do not have a higher probability of occurrence of these biomarkers, exposures less than $0.25 \mathrm{ppm}$ over a 40 year working lifetime may not represent a significant risk.

However, additional confounding factors may need to be considered. For example, VC workers who experience both mutations may do so because they are somehow extraordinarily susceptible to the mutagenic effects of the exposure. Other studies have demonstrated that VC workers with genetic polymorphisms in the enzymes responsible for the metabolism of VC (CYP 2E1 and ALDH-2), which could lead to an increased formation of DNA adducts at any given exposure level, are more likely to demonstrate the effects of genetic damage in terms of a significantly increased frequency of sister chromatid exchanges compared to VC workers with normal variants of these enzymes (Wong et al. 1998). In fact, in the present cohort thus far, one of the workers with the presence of both oncoprotein biomarkers has been found to have the susceptible variant of the CYP 2E1 gene. In addition, it is possible that genetic polymorphisms in the enzymes responsible for repair of DNA adducts could have a similar effect. Studies are underway to examine these possibilities. Eventually, it may be possible to perform the risk assessment for VC exposure on the basis of the presence of oncoprotein biomarkers stratified by genetic susceptibility due to VC metabolism and/or DNA repair, which would represent a considerable refinement over current approaches.

At the level of the individual worker, however, identification of an elevated risk for cancer based on the occurrence of mutant biomarkers and a susceptible genotype is of little significance unless secondary prevention can be employed to help minimize the risk. One such approach that might be useful with these workers would be to try to correct the specific functional deficits produced by the VCinduced mutations. For example, the mutations in p53 from VC cause common conformational changes in the protein that account for its loss of function for tumor suppression and programmed cell death (apoptosis) (Brandt-Rauf et al. 1996). We have shown that mutant p53 in cancer cells can be induced to revert to normal function through interaction with small peptides that represent sequences from the 
regulatory domain of the protein, resulting in apoptosis of the cancer cells, but that these peptides have no adverse effect on nonmalignant human cell lines containing wild-type p53 (Kim et al. 1999). Such peptides could form the basis for chemotherapy for individuals with tumors that contain p53 mutations and as chemoprophylaxis for individuals who are at risk for the development of such tumors due to the occurrence of p53 mutations from their exposures, such as those VC workers who are positive for the biomarker. High-risk workers could thus be identified and treated early, preventing the development of future cancers. Therefore, ultimately, molecular biomarkers could be useful in contributing to group risk assessment to refine permissible exposure limits improving future primary prevention and in individual risk assessment to identify workers for potential chemoprophylaxis for secondary prevention of the effects of such carcinogenic exposures.

\section{ACKNOWLEDGMENT}

This work was supported in part by grants from the U.S. National Cancer Institute (R01-CA69243 and T32-CA09529) and the U.S. Environmental Protection Agency (R-825361 and R-826685), and the U.S. National Institute for Occupational Safety and Health (R01-OH04192).

\section{REFERENCES}

ATSDR (Agency for Toxic Substances and Disease Registry). 1997. Toxicological Profile for Vinyl Chloride (Update). DHHS Publication No. TP-92/20. U.S. Department of Health and Human Services, Public Health Service, Atlanta, GA, USA

Barbin A and Bartsch H. 1986. Mutagenic and promutagenic properties of DNA adducts formed by vinyl chloride metabolites. In: Singer B and Bartsch H (eds), The Role of Cyclic Nucleic Acid Adducts in Carcinogenesis and Mutagenesis, pp 345-58. International Agency for Research on Cancer, World Health Organization, Lyon, France

Brandt-Rauf PW, Chen JM, Marion MJ, et al. 1996. Conformational effects in the p53 protein of mutations induced during chemical carcinogenesis: Molecular dynamic and immunologic analyses. J Protein Chem 15:367-75

Brandt-Rauf PW, Luo JC, Cheng TJ, et al. 2000. Mutant oncoprotein biomarkers of vinyl chloride exposure: Applications to risk assessment. In: Anderson D, Karakaya AE, and Sram RJ (eds), Human Monitoring after Environmental and Occupational Exposure to Chemical and Physical Agents: Proceedings of the NATO Advanced Study Institute, pp 243-8. IOS Press, Amsterdam, The Netherlands

DeVivo I, Marion MJ, Smith SJ, et al. 1994. Mutant c-Ki-ras p21 protein in chemical carcinogenesis in humans exposed to vinyl chloride. Cancer Causes Control 5:273-8

Hattis D and Silver K. 1993. Use of biomarkers in risk assessment. In: Schulte PA and Perera FP (eds), Molecular Epidemiology Principles and Practice, pp 251-73. Academic Press, San Diego, CA, USA

Hollstein M, Marion MJ, Lehman T, et al. 1994. P53 mutations at A:T base pairs in angiosarcomas of vinyl chloride-exposed factory workers. Carcinogenesis 15:1-3

Husgafvel-Pursiainen K, Kannio A, Oksa P, et al. 1997. Mutations, tissue accumulations and serum levels of p53 in patients with occupational cancers from asbestos and silica exposure. Environ Mol Mutagen 30:224-30

Li Y, Marion MJ, Asherova M, et al. 1998. Mutant p21ras in vinyl chloride-exposed workers. Biomarkers 3:433-9 
Luo JC, Liu HT, Cheng TJ, et al. 1998. Plasma asp13-Ki-ras oncoprotein expression in vinyl chloride monomer workers in Taiwan. J Occup Environ Med 40:1053-8

Luo JC, Liu HT, Cheng TJ, et al. 1999. Plasma p53 protein and anti-p53 antibody expression in vinyl chloride monomer workers in Taiwan. J Occup Environ Med 41:521-6

Kim AL, Raffo AJ, Brandt-Rauf PW, et al. 1999. Conformational and molecular basis for induction of apoptosis by a p53 C-terminal peptide in human cancer cells. J Biol Chem 49:34924-31

Marion MJ, Froment O, and Trepo C. 1991. Activation of Ki-ras gene by point mutations in human liver angiosarcoma associated with vinyl chloride exposure. Mol Carcinogen 4:450-4

Smith SJ, Li Y, Whitley R, et al. 1998. Molecular epidemiology of p53 protein mutations in workers exposed to vinyl chloride. Am J Epidemiol 147:302-8

Swenberg JA, Fedtke N, Ciroussel F, et al. 1992. Etheno adducts formed in DNA of vinyl chloride- exposed rats are highly persistent in liver. Carcinogen 13:727-9

Tornqvist $\mathrm{M}$ and Landin HH. 1995. Hemoglobin adducts for in vivo dose monitoring and cancer risk estimation. J Occup Environ Med 37:1077-85

Wong RH, Wang JD, Hsieh LL, et al. 1998. Effects on sister chromatid exchange frequency of aldehyde dehydrogenase 2 genotype and smoking in vinyl chloride workers. Mut Res 420:99- 107 
Copyright $\odot 2002$ EBSCO Publishing 